\title{
Application Portfolio Profiling and Appraisal as Part of Enterprise Adoption of Cloud Computing
}

\author{
Venkata Naga Satya Surendra Chimakurthi \\ Technical Manager - Projects, EBA - Ventures - Digital Asset Management (DAM), Cognizant Technology Solutions, Dallas, USA \\ *Corresponding Contact: \\ Email: chvnssurendra@gmail.com
}

Manuscript Received: 29 Sept 2019

Revised: 12 Dec 2019

Accepted: 28 Dec 2019

\begin{abstract}
Computation resources have never been more affordable, powerful, or readily available than they are now, thanks to the Internet and the rapid advancement of processing and storage technologies. In response to this new trend in technological development, a brand-new computer paradigm is known as "cloud computing" has emerged. Resources are made available to end-users as general utilities that can be rented and released on-demand over the Internet. More and more corporate processes are being moved to the cloud as firms experience using the technology. Cloud adoption is substantially more complicated than we first thought when it comes down to data management, system integration, and managing various cloud providers. As cloud migration has grown more common, a number of have been plagued by failed application modernization initiatives or migration. However, many firms worldwide are finding that cloud computing is more expensive and more difficult to implement than they expected. Is cloud computing still up to the task in this scenario? Failures are largely due to a lack of planning in the early stages of identifying which legacy applications are best suited for private or public cloud deployment, resulting in cost and time overruns. Application portfolios need deliberate decision-making to reduce the risk of overruns and delays in migration. Consequently, a rough estimate of cloud operating costs (ROM) is required for inclusion in the planning phase of IT governance. ROM cloud run costs must first be gathered through an automated discovery process, followed by a quantitative analysis. Accordingly, to assist in making an informed decision about whether or not to migrate legacy applications, we propose two methods: (1) gathering initial data through Application Portfolio Profiling (APP), and (2) performing Application Portfolio Assessment (APA). An Enterprise Cloud Computing Considerations Framework (CCCC) framework is used to help a corporation decide which legacy applications should be moved as part of the adoption process for Cloud Computing. In light of the shift in business models, infrastructure requirements and other issues that arise when cloud computing is implemented, this decision is crucial.
\end{abstract}

Key Words: Cloud Migration, Enterprise Cloud Computing Adoption, Application Portfolio Assessment, Application Portfolio Profiling 


\section{INTRODUCTION}

Recently, cloud computing has evolved as a new paradigm for delivering and hosting Internet services. For business owners, cloud computing is attractive because it eliminates the need for users to prepare in advance for provisioning and allows organizations to start small and add resources as service demand increases. However it's important to note that even while cloud computing has enormous potential for the I.T. business, the field is still developing, and there are numerous challenges to be worked out. According to market data, cloud computing adoption is on the rise; for companies with many legacy applications that need to be moved into the cloud, obtaining a Rough Order of Magnitude (ROM) of cloud run and migration expenses would be a useful tool in assessing the feasibility and affordability of cloud computing. Insufficient planning or governance is the leading cause of cost and time overruns, and as a result, there are no mitigation methods for cloud risk or alignment with company goals. In order to make a business case for cloud computing it is necessary to deal with a large number of stakeholders, which makes it difficult to put together a business plan before implementing cloud services (Hassanalieragh et al., 2015). Since significant capital investments have traditionally been the impetus for business cases, it's easy for companies to think that they won't need to make any more now that some commercial cloud models allow customers to gain the needed capabilities immediately. Even while the number of businesses moving to the cloud is growing, few have developed a strategy for estimating the total amount of money that will be required before moving to the new operating model, which goes beyond the migration itself (Chen \& Sion, 2011).

\section{LITERATURE REVIEW}

Studies on enterprise Cloud Computing adoption frameworks show that several attempts have been made at decision support models for cloud adoption, as shown by an examination of current research and industry publications (Chang et al., 2016). It has been shown that cloud migration frameworks, techniques, and cost models are scattered and fragmented, requiring new scientific approaches to research on the topic of cloud migration (Talukder et al., 2010). The primary ways to live migration of virtual machines are discussed in the study of (Choudhary et al., 2017). Particular attention will be given to a study of the most up-to-date optimization strategies for creating live V.M. migrations that adhere to memory migration best practices. To understand the advantages and disadvantages of these strategies, it reviews, discusses, analyses, and contrasts them. Live migration for virtual machines is an area where further research is needed, and this paper identifies the areas that need to be addressed. Though cloud migration is widely used these days, there are still difficulties in adopting it. In the article (Opara-Martins et al., 2014), researchers have examined the most important drivers affecting enterprise adoption of cloud computing. There are several cloud frameworks already in existence that have been analyzed in this research. According to the identified variables, a theoretical framework for cloud adoption has been developed (Chimakurthi, 2018). The results show that cloud adoption is positively influenced by technological, organizational, and certain external variables.

Researchers looked into how much computing power should be devoted to moving enterprise apps to the cloud at one time (Williams, 2018). We begin by defining a generalization of the utility maximization model for resource allocation in the migration of enterprise applications. The non-strict convexity and non-separation of the generalization-form optimization problem make it challenging to solve. The generalization-form model is studied using the successive approximation method, and a resource allocation mechanism based on gradients is presented 
to address the approximation issues. Using cloud computing, organizations can take advantage of scalability, agility, high availability, and cost savings. Migrating old systems to the cloud makes sense for many businesses because of these advantages (Williams \& Scott, 2019). However, due to their design, these older apps must be migrated using a tried-andtrue method that carries no risk. For legacy systems moving to the cloud, only a few models or frameworks have been presented. Many of these cloud adoption strategies and models focus on the generic phases and procedures for moving apps and data to the cloud. This is not to say that all of these models are the same. The study of (Pahl \& Xiong, 2013) examines the challenges of moving legacy apps to the cloud, as well as the various methods and models that have already been offered.

\section{Cloud Computation in EnTERPRise}

It is becoming increasingly common for governments around the world to promote the use of cloud computing services as a means of increasing the productivity, innovation, and agility of the digital economy. Revenues from worldwide cloud computing climbed $28 \%$ to $\$ 110$ billion in 2015, according to industry research firm Gartner.

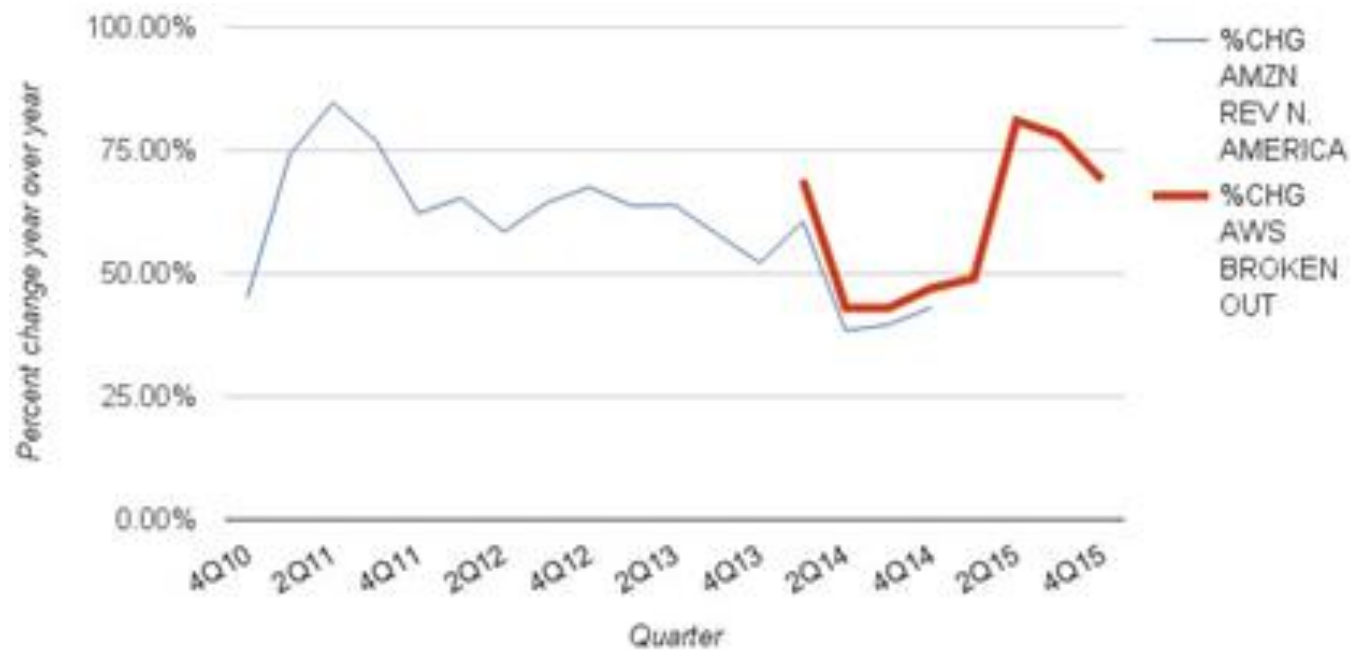

Figure 1: Growth in AWS revenue

Without a defined business case, some firms have effectively implemented cloud computing. The most common cloud computing traps, such as unexpected costs or delays in migrating legacy applications to the cloud, can be avoided by performing a thorough business case and cost analysis in advance. An enterprise's current expenses may be surpassed by the expenses of a rushed or unprepared transition to the public cloud and PAYG usage business model. Internet storage and retrieval in the form of an Approaching cloud adoption with an understanding of the expected operational cost and public investment required for application transfer is critical to avoid being caught off guard, untrained, or reluctantly investing in the necessary investments. All service types are included in the ROM estimations as part of the company's cloud migration plan, which is part of an overall IT governance plan. (Montenegro et al., 2017).

It is possible to make tactical cloud migration decisions for certain apps, but this does not ensure the best cloud migration option for a whole enterprise's portfolio because migration 
and ongoing expenses are not frequently taken into account (Chimakurthi, 2017a). An organized cloud strategy and framework must be in place. An enterprise strategy and an application portfolio assessment must be used to determine the readiness of an application for the cloud when moving outdated apps.

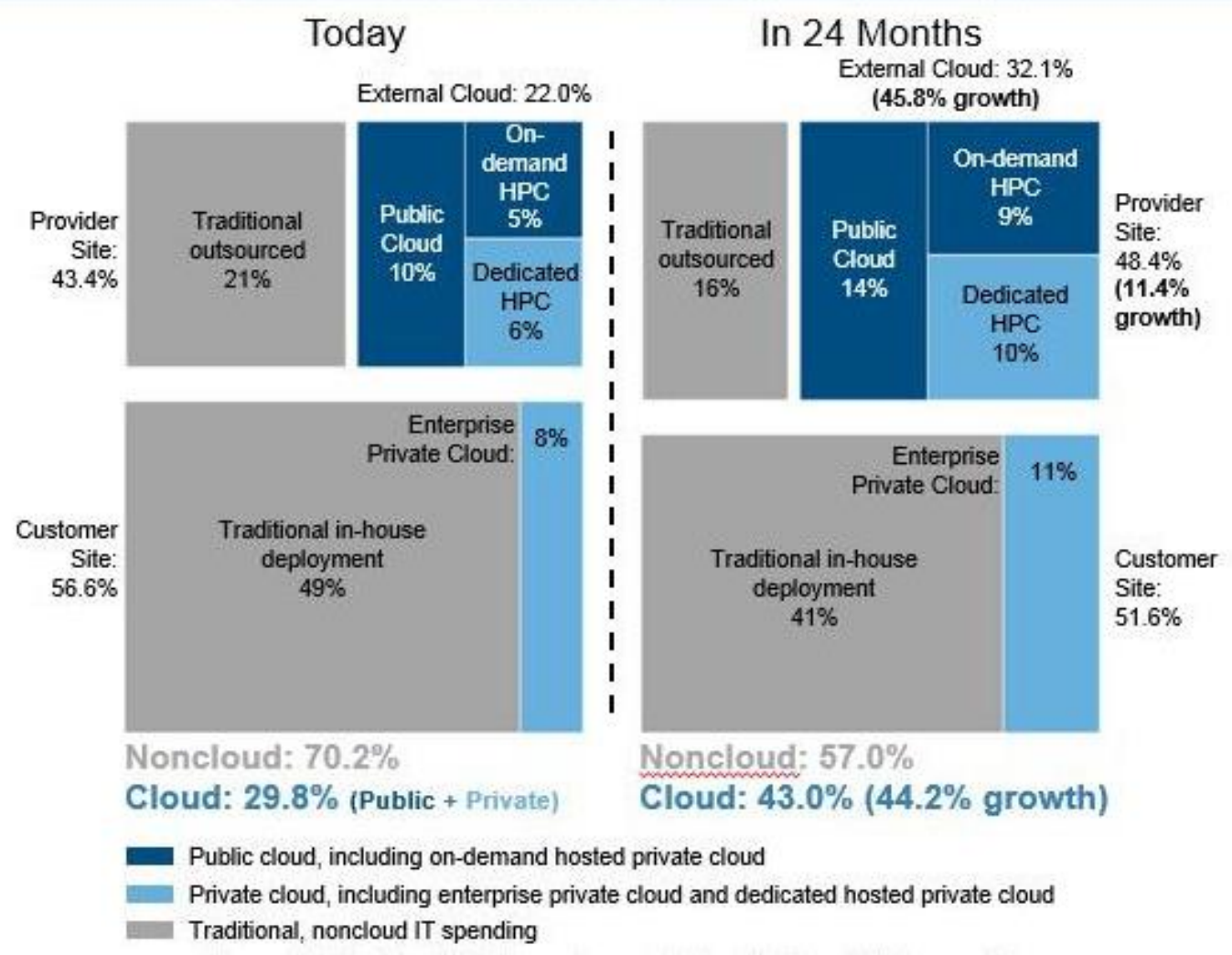

Figure 2: I.T. Infrastructure Redistribution - Public and Private Cloud

Starting with apps that perform comparable functions (such as web servers), the most sophisticated applications should be left till the last when an organization is considering Cloud Computing adoption. Rework can be avoided by using an enterprise approach, which is in line with the business strategy (Talukder et al., 2010) and to ensure that all of the company's applications function together. It is also more compelling to make the business case for cloud computing adoption at enterprise size when the benefits of converting and eliminating complexity, and hence expense, from the current I.T. infrastructure, are taken into account.

SaAS and private cloud should all be factored into any 3- or 5-year cloud platform operating cost projection model. Public cloud migration frameworks and TCO calculators are currently available (Pahl \& Xiong, 2013). It's still possible to determine affordability by evaluating the application portfolio and estimating the expenses of Cloud Computing adoption at the ROM level. 


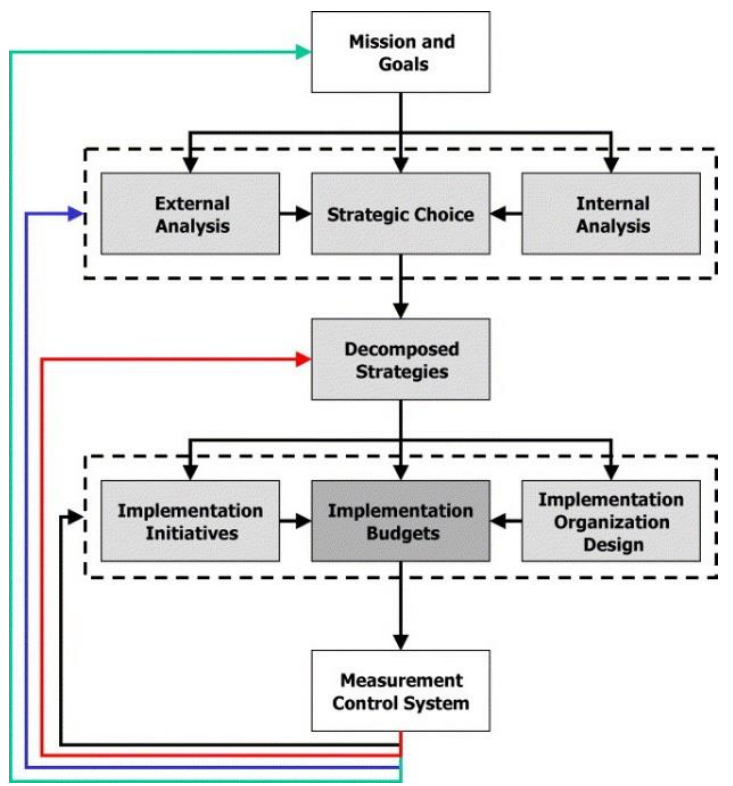

Figure 3: Enterprise Strategic and IS/IT system
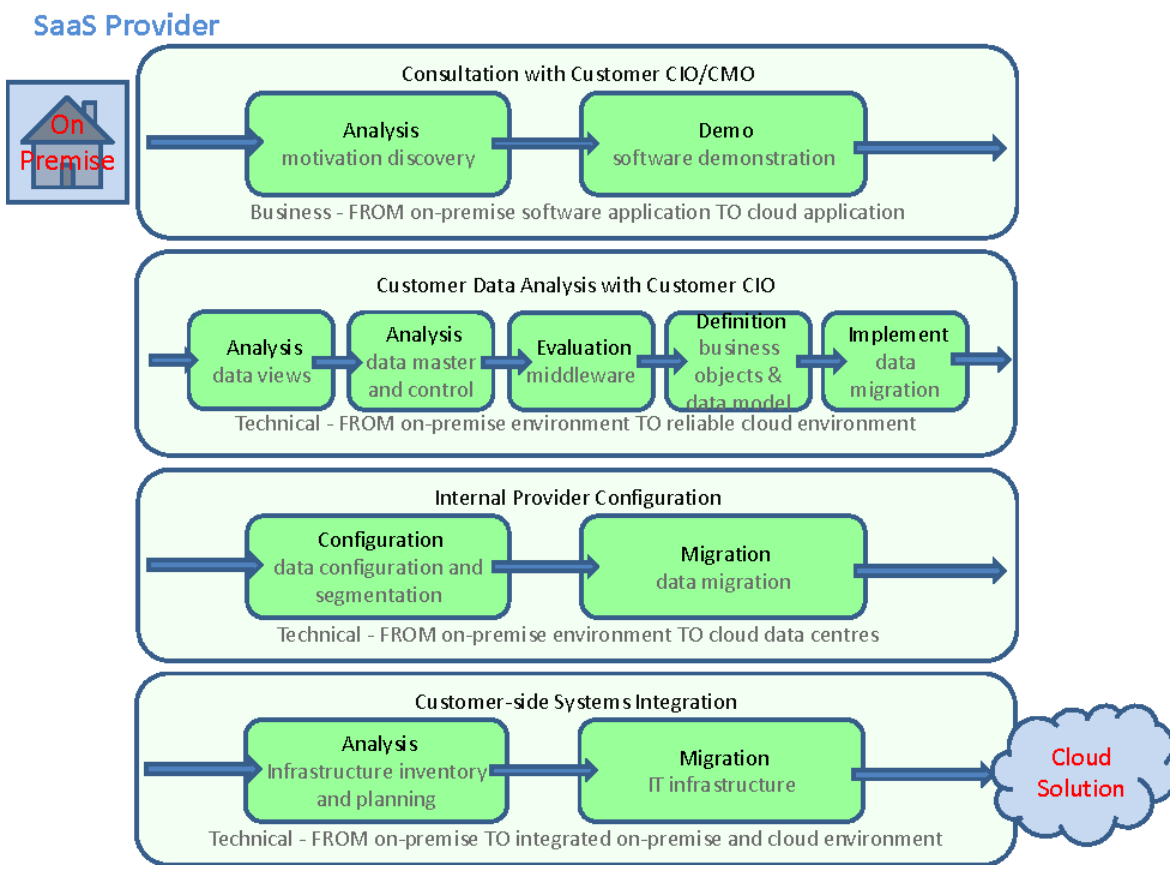

Figure 4: PaaS Migration - Process Perspective

We've also taken the approach of standardizing cloud service provider calculators so that they can account for things like adoption costs, changes in commercial models, and new players entering the market. It is possible to examine the cost-effectiveness of hybrid cloud computing and the amount of money needed to migrate legacy applications in a timely and cost-effective manner, thanks to the ROM expenses created. 


\section{Enterprise Application Migration Bandwidth Allocation}

Effective bandwidth resource allocation can assist cloud vendors avoid network congestion while also enhancing the performance of enterprise applications. The shorter the time it takes for a cloud data center to execute parallel computing activities, the more bandwidth a company can obtain. A lack of bandwidth resources can result in a delay in transmission or a longer task completion time for enterprise users. In other words, faster cloud migration and more company satisfaction go hand in hand when consumers have access to additional bandwidth resources.

When transferring corporate programs to the cloud, bandwidth is allocated to each component of the application in each virtual machine managed by a physical computer. The corresponding resource is then allocated to cloud data center virtual machines. As a result, the total bandwidth resources $z s=\sum_{\mathbf{s} \in \mathbf{S}(\mathbf{l})} \quad \sum_{\mathbf{l} \in \mathbf{L}(\mathbf{s})} x l s r$ for every application $\mathrm{s} \in \mathrm{S}$ is used.

$\sum_{\mathbf{s} \in \mathbf{S}(\mathbf{l})} \sum_{\mathbf{r} \in \mathbf{R}(\mathrm{s})} \leq \mathrm{Cl}, \forall \mathrm{l} \in \mathrm{L}$ is satisfied since no link can take on more work than it can handle due to the inherent limitations of each link. Each application I has a load of Ds. There are a number of considerations for enterprise users when migrating local apps to the cloud, such as ensuring the migration satisfaction of enterprise users. Application transmission in the cloud data center must be completed by a specified deadline, indicated by $\mathrm{T}$; the real time is $\sum \mathrm{s} \in \mathrm{SDs} / \mathrm{zs}$. When it comes to migrating enterprise apps to the cloud, bandwidth allocation can be described as the transmission time optimization problem $\mathrm{M}$.

$M: \max T-\sum_{\mathrm{s} \in \mathrm{S}} \frac{\mathrm{Ds}}{\mathrm{ZS}}$

subject to $\sum_{\mathrm{s} \in \mathrm{S}(\mathrm{l})} \sum_{\mathrm{l} \in \mathrm{L}(\mathrm{s})} x^{l} \mathrm{~s} r=\mathrm{zs}, \forall \mathrm{s} \in \mathrm{S}$

$\sum_{s \in S(l)} \sum_{r \in R(s)} \leq \mathrm{Cl}, \forall \mathrm{l} \in \mathrm{L}$

over $\underset{x}{\stackrel{l}{s} r} \geq 0, \mathrm{~s} \in \mathrm{S}, \mathrm{r} \in \mathrm{R}, \mathrm{l} \in \mathrm{L}$.

Maximum transmission time difference is dependent on total bandwidth allocation obtained by each application, which is the objective of bandwidth allocation issue $\mathrm{M}$ for application components. As a result, the issue $\mathrm{M}$ pertains to the distribution of bandwidth resources throughout the diverse set of resources $\mathrm{z}^{\mathrm{s}}$.

\section{Cloud Computing as a Foundation for Business Strategy}

CCCC presents a complete method for analyzing whether or not cloud computing adoption in enterprises is affordable, and we suggest it to address the difficulties listed above. Using this approach, you can get a decent estimate of cloud expenses while also taking into account the amount of time it takes to perform the evaluation. Cloud Decision Framework (CDF) is a decision-support tool. Now, organizations can use CDF to make decisions about a portfolio of apps. (Ramchand et al., 2017). A single application can be evaluated using the CDF, but a collection of apps can be evaluated using the CCCC framework, which considers the interdependencies among them.

I.T. governance requires an early study of the cloud operating costs for each application portfolio in order to avoid overruns or delays in the process. Ideally, baseline application data should be gathered automatically, and quantitative analysis of the applications should be 
conducted. A quantitative analysis of applications (APA) and an Application Portfolio Profiling (APP) are proposed to guide the migration of older applications from their current environment. It is possible to classify the adoption of Enterprise Cloud Computing as a strategic decision rather than a tactical one for a specific application. To stay relevant, companies in every sector are scrambling to adopt cutting-edge business concepts as rapidly as possible. While monolithic workloads are predictable, unexpected usage volume and geographic dispersion necessitate infrastructure flexibility, performance, and elasticity. Already, several businesses are experimenting with Cloud Computing (Chimakurthi, 2017b) with varying degrees of success. As part of the CCCC framework, an application portfolio's financial viability and alignment with the business plan are assessed as part of the FVA. This offers a picture of cloud running costs.

Table 1: Preliminary Revenue Estimates for Public Cloud Services Worldwide (Billions of U.S. Dollars) 2016)

\begin{tabular}{|l|c|c|c|c|}
\hline & $\mathbf{2 0 1 4}$ & $\mathbf{2 0 1 5}$ & $\mathbf{2 0 1 6}$ & $\mathbf{2 0 1 7}$ \\
\hline Cloud Application Infrastructure Services (PaaS) & 2.3 & 3.1 & 4.0 & 4.9 \\
\hline Cloud Application Services (SaaS) & 3.1 & 4.1 & 5.4 & 6.8 \\
\hline Cloud System Infrastructure Services (IaaS) & 7.0 & 9.7 & 12.5 & 15.4 \\
\hline
\end{tabular}

PaaS = platform as a service; IaaS = infrastructure as a service; SaaS = software as a service

There are several factors to consider when choosing between public or private cloud computing for legacy applications, including cost and suitability, which necessitates an early assessment. For a company to successfully migrate its legacy applications to the cloud, it must first identify and account for the cloud's hidden expenses. The true cloud operation costs can become unaffordable if these fees get too high. These expenses can be substantial for important applications and services that are critical to the success of a company. Because of this, an application portfolio-aligned cloud strategy necessitates a systematic structure. That, in turn, adds to the definition of the cloud target state for a hybrid cloud result (Mitropoulou et al., 2016).

\section{Comparison of Cloud Adoption FramewORKS}

A survey of recent literature and industry publications (Chang et al., 2016) on enterprise Cloud Computing adoption frameworks demonstrates that numerous attempts have been made at decision support models. An evaluation of number of Cloud Adoption and Migration studies (Verdejo \& Baars, 2013) also indicated that cloud migration methods, and expenses models are scattered, necessitating new scientific approaches to cloud migration research. For an application portfolio's affordability and suitability, this paper's new CCCC framework approach assesses it from a technical and financial standpoint. When migrating large-scale and sophisticated legacy systems that underpin an organization's key business activities, a systematic approach becomes increasingly vital.

Technical and economic feasibility must be considered prior to migrate an old application using the ARTIST Methodology and Framework (Kossmann et al., 2010). Cloud computing's hidden costs are not taken into account when calculating projected cloud run costs, nor does it assist in assessing whether an application should be placed in a private or public cloud. It has a limitation of looking at each application separately, which can lead to delays and cost overruns. As a result, an application's performance and stability may be compromised as the cloud platform, and any legacy apps that have yet to be migrated are integrated more slowly. It is also possible to make use of the cloud's elasticity and performance by using a model for moving existing on-premises applications to it. 
Organizational readiness, security readiness, skill availability, and telecom infrastructure availability are all part of the cloud computing adoption paradigm presented in. Alternately, a government-specific cloud migration strategy (Chang et al., 2016) focuses on technological criteria. Each of these factors is given equal weight, and a final score is calculated based on that weight to help determine whether or not public cloud computing is appropriate. Choosing a cloud platform and determining the FVA for cloud run and migration costs are both part of the IT governance process, but the CCCC framework offers an integrated approach that differs from both of these approaches in important ways. This illustrates the existing dispersion in cloud adoption research. As an alternative, only older apps can be evaluated for migration to the cloud-based on non-functional needs (Verdejo \& Baars, 2013).

\section{Cloud Decision FrameWork}

The Cloud Decision Framework uses Rule-based Reasoning (RBR) and Case-based Reasoning (CBR) to recommend an application for a cloud platform (see Figure 5). Before a TPR can be provided, a business sponsor employing the RBR-based decision framework must generate a complete set of criteria and classifications. In this case, it's the norm. Since requirements gathering hasn't concluded, it's unlikely that a firm SME will know all of the criteria in the framework for applicability or values. RBR and CBR assist the cloud adopter in eliciting business requirements and cloud platform suggestions when the cloud adopter is not sure about requirements or lacks complete information. Finding similar case histories can be done using a set of established criterian that can then be used to propose which one is the most appropriate for adoption.

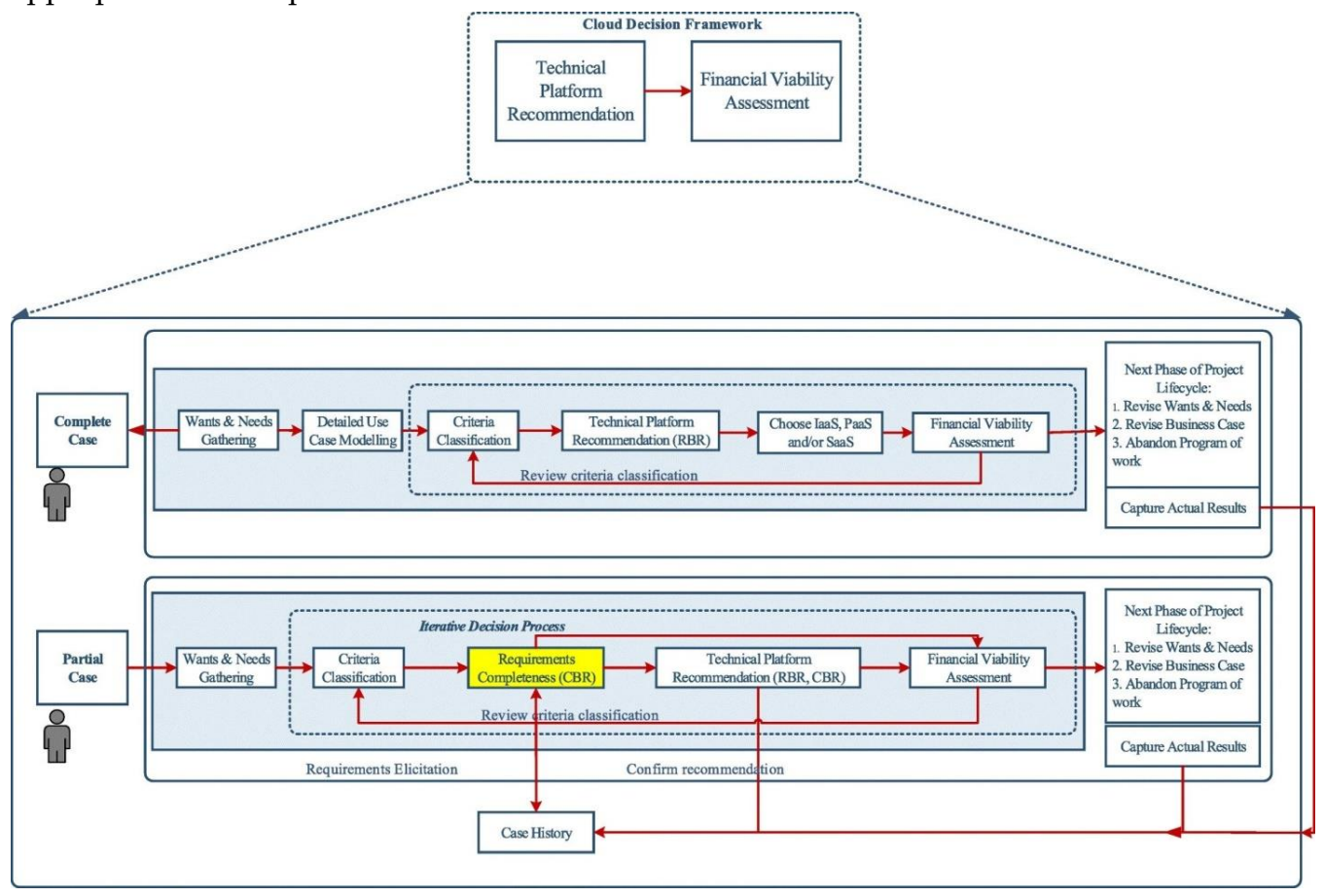

Figure 5: Cloud Decision Framework using CBR and RBR

An SME must establish a business case migration and then choose from a variety of alternatives, such as traditional IT, private or public clouds, or a combination of these. This is 
above in Figure 5. Iterative decision-making occurs in three stages: There are a number of requirements that are either "required" or "optional" for a cloud user or small to mediumsized business (SME). As a result of the CC entry, an organization's computing requirements are analyzed using TPR and FVA to determine whether a public or private cloud is the best option.

\section{Cloud Computing Considerations for Companies (CCCC) Framework}

Tactical support for making decisions about whether to use public or private IaaS is provided by the CDF shown in Figure 6, which helps guarantee that the Cloud Computing platform is both functional and financially sustainable. When evaluating a portfolio of applications, however, this approach is flawed since, following an IaaS selection, it is necessary to verify that the location of each application will not have an adverse effect on its performance or cost. In order to make a 'Data Placement' architectural decision, more information is needed throughout the application portfolio. As a result of egress fees or poorly performing software, poor data location decisions can lead to unexpectedly large data expenditures in the future. If you use a hybrid cloud application, your data is likely to move between a private and public storage environment, resulting in cloud and network expenses. A Cloud Computing payment might increase by thousands of dollars a month because of the cost of moving data between the data center and the cloud platform.

Taken at the planning phase of enterprise Cloud Computing deployment, an application portfolio approach frequently exposes these issues:

- Application silos are recognized for each business section with a bulk of linkages that must be taken into consideration to make sure that end users are not influenced throughout the migration. A wave is an effective "segmentation" or self-contained work activity with a virtual or physical server-to-application grouping.

- Brittle and unstable point-to-point integration relies on fixed settings that must be modified during migration.

As a result, the CCCC framework, shown in Figure 5, is an extension of the prior CDF. All of the above are supported by the CCCC framework.

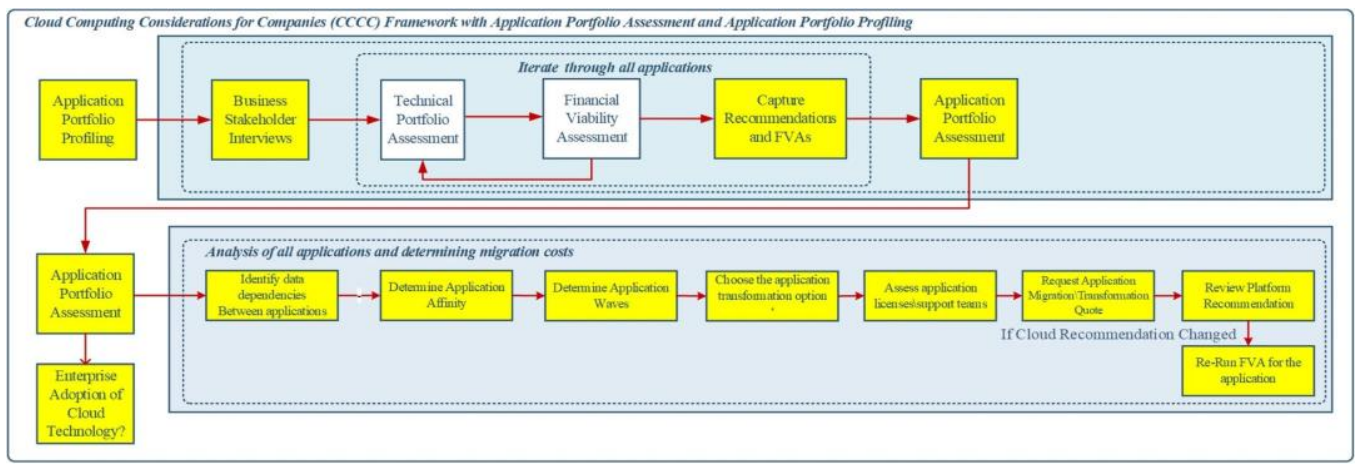

Figure 6: CCCC Framework

- It is feasible to use the Application Portfolio Decision Process (APP) and the Application Portfolio Analysis Process (APAP) to identify old applications that are economically and technically reliable for cloud computing while also taking account and allowing for their interrelations when cloud run costs and forecasting migration (APA). 
- $\quad$ Each application in the portfolio is analyzed using an APA following interviews with relevant stakeholders. The APA is built on the foundation of the data collected throughout this data collection procedure.

- Each application's dependencies, affinity, role, and cost-saving options such as consolidating managed services and service desks are identified this phase.

- $\quad$ Businesses should consider expanding their use of tactically optimized decision-making in order to take use of cloud computing on a larger scale.

- Use the CCCC framework's output to examine the hidden expenses of cloud computing and the affordability of cloud adoption, allowing stakeholders to compare the pricing of private and public clouds for each application.

- Determine if the savings from moving and transforming apps to a Hybrid Cloud are sufficient to cover the costs of the migration and transformation.

- For determining whether or not a Hybrid Cloud migration or transformation is financially viable, the business case formula is as follows: Costs of compute/network/storage as-is vs. projected IaaS costs for public and private clouds in years 1, 2, 3, 4, and 5. Since cloud computing costs can be recovered in years one, two, and three, an enterprise's move to cloud computing is deemed feasible and even advised by many experts.

\section{Application Portfolio Profiling}

Figure 6 shows the CCCC framework, which begins with the APP, including the following steps:
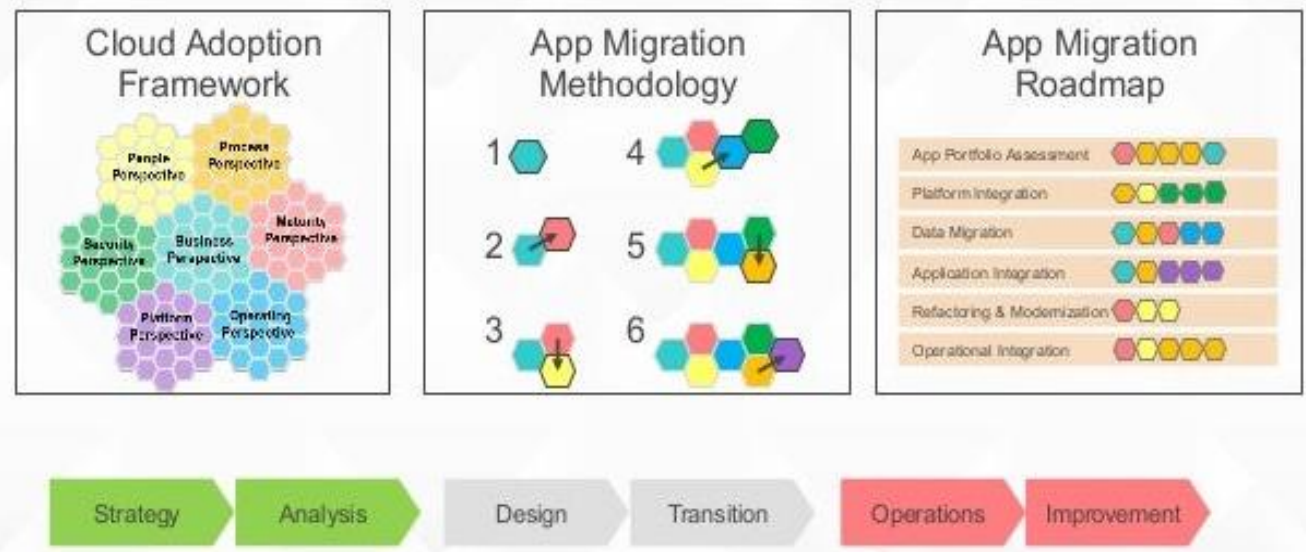

Figure 7: Building the App Migration Roadmap

\section{Interviewing business stakeholders}

When an application is migrated, relevant stakeholders are interviewed in order to get information about the business plans for the application and critical success indicators, such as compliance with availability and performance of Service Level Agreements (SLA).

\section{Data collection}

The titles, imprints, and regularity of use of business applications are all collected here. Data can be collected automatically using IBM's Tivoli Application Discover BMC Helix Discovery and Dependency Management (COTS). 


\section{Filtering application}

A list of applications that should be retired because they no longer serve a strategic purpose is compiled. For instance,

- Applications that aren't acceptable or unnecessary in a cloud computing architecture are excluded from the evaluation based on their software licensing agreements and operating system selection.

- Applications that are no longer of strategic importance or are scheduled for obsolescence are not examined.

For strategic applications, key stakeholder interviews are conducted to establish the migration technique and the degree of application transformation, if necessary. For example, after discovering and profiling an application's data and completing the data discovery process, the attention might shift to verifying the data and deciding whether to migrate the application in its current form or alter it.

\section{Capturing TPRs and FVAs}

TPR and FVA are tallied for each application involved in the assessment by the cloud adopter or small and medium-sized enterprise (SME).

\section{Application Portfolio Assessment}

Tasks such as this are carried out next in APA:

\section{Identifying data dependencies among applications}

It's the data interconnections that drive cloud software data management, which affect requirements like data integration, data migration, and referencing data management.

\section{Identification of application affinity}

Identifying groups of related apps that should be moved in a logical fashion is a part of this stage. To determine Application Affinity, follow these steps:

- Checking and verifying the present state of the physical and virtual server environment.

- The sizing of the environment to be targeted.

- Application linkage/communications identification and establishment from an "as-is" and a "to-be" environment.

- Using Application Affinity and identifying target architectural configurations to build an application migration wave strategy to minimize migration risk. This entails creating a functional map of all applications in the portfolio and then assigning each application to a grouping inside that map, resulting in a migration wave.

\section{Choosing a transformation option for an application}

In this stage, you'll figure out how much work it will take to make the switch to the recommended cloud platform. Tasks such as these are among the examples:

- $\quad$ Making the most of Cloud Computing's transformative potential.

- $\quad$ Security designs that can be implemented include encryption, zoning maps, and access to the environment, procedures, and processes by professional services partners. 


\section{Evaluation of software support and license teams}

When assessing software licenses and/or application support teams, the ones that can be retired, excess to requirements, or consolidated are identified in this phase of the process.

\section{Request a quote for the migration or transformation of an application.}

Applications that have been identified as being appropriate for Cloud Computing are requesting quotes for migration and transformation.

\section{Recommendation review of a platform}

According to their application wave allocation, applicants' TPRs are updated. In cases where the TPR changes, the FVA should be recalculated and the combined expenses updated correspondingly (Lam, 2017).

\section{CONCLUSION}

Slash development cycle speeds while increasing capacity on-demand to cut operations and infrastructure costs in the cloud. As the popularity of cloud computing and the migration of legacy applications increases, companies will need a strategy for influencing IT governance choices through the use of cloud adoption. A lack of research on cloud acceptance and legacy application migration was observed because many existing options were only explored from a technical or economic perspective. Consequently, here we describe a method for acquiring an app portfolio's running and migration expenses at the planning phase of a cloud migration utilizing APP and an APA before undertaking quantitative analysis of the apps. Both APP and APA are now part of our cloud decision architecture. In this approach, organizations may make educated decisions about which legacy apps should be migrated to the cloud as part of their enterprise cloud computing adoption by considering both technical and financial issues. We put the CCCC framework to the test in a real-world setting, and the results were encouraging.

SLA Manager's build and run expenses as well as the standards necessary for consistency across environments and management tools will be assessed and included in the next phase of our work to minimize the risk of increasing the complexity of managing federated or hybrid cloud. Further validation of our framework will be done using further V.A. case studies, which will help us better understand the framework and how it should be used. What we're going to do next is look at how the culture and skill sets in a company's workforce might affect training requirements, and how that can impact both on-shore and off-shore personnel.

\section{REFERENCES}

Chang, V., Kuo, Y.-H., \& Ramachandran, M. (2016). Cloud computing adoption framework: A security framework for business clouds. Future Generation Computer Systems, 57, 24 41. https://doi.org/10.1016/j.future.2015.09.031

Chen, Y., \& Sion, R. (2011). To cloud or not to cloud? Proceedings of the 2nd ACM Symposium on Cloud Computing. SOCC '11: Proceedings of the 2nd ACM Symposium on Cloud Computing, Article No.: 29, 1-7. https://doi.org/10.1145/2038916.2038945

Chimakurthi, V. N. S. S. (2017a). Cloud Security - A Semantic Approach in End to End Security Compliance. $\quad$ Engineering International, 57106. https:// doi.org/10.18034/ei.v5i2.586 
Chimakurthi, V. N. S. S. (2017b). Risks of Multi-Cloud Environment: Micro Services Based Architecture and Potential Challenges. ABC Research Alert, 5(3), United States. https://doi.org/10.18034/abcra.v5i3.590

Chimakurthi, V. N. S. S. (2018). Emerging of Virtual Reality (VR) Technology in Education and Training. Asian Journal of Humanity, Art and Literature, 5(2), 157 166. https://doi.org/10.18034/ajhal.v5i2.606

Choudhary, A., Govil, M. C., Singh, G., Awasthi, L. K., Pilli, E. S., \& Kapil, D. (2017). A critical survey of live virtual machine migration techniques. Journal of Cloud Computing, 6(1). https://doi.org/10.1186/s13677-017-0092-1

Hassanalieragh, M., Page, A., Soyata, T., Sharma, G., Aktas, M., Mateos, G., ... Andreescu, S. (2015). Health Monitoring and Management Using Internet-of-Things (IoT) Sensing with Cloud-Based Processing: Opportunities and Challenges. 2015 IEEE International Conference on Services Computing, 285-292. https://doi.org/10.1109/scc.2015.47

Kossmann, D., Kraska, T., \& Loesing, S. (2010). An evaluation of alternative architectures for transaction processing in the cloud. Proceedings of the 2010 ACM SIGMOD International Conference on Management of Data, 579-590. https:// doi.org/10.1145/1807167.1807231

Lam, R. (2017). Taking stock of portfolio assessment scholarship: From research to practice. Assessing Writing, 31, 84-97. https://doi.org/10.1016/j.asw.2016.08.003

Mitropoulou, P., Filiopoulou, E., Michalakelis, C., \& Nikolaidou, M. (2016). Pricing cloud IaaS services based on a hedonic price index. Computing, 98(11), 1075-1089. https://doi.org/10.1007/s00607-016-0493-x

Montenegro, C., de la Torre, A., \& Nuñez, N. (2017). Integrated IT Governance and Management Model: Evaluation in a Developing Country. Advances in Intelligent Systems and Computing, 71-81. https://doi.org/10.1007/978-3-319-69341-5 7

Opara-Martins, J., Sahandi, R., \& Tian, F. (2014). Critical review of vendor lock-in and its impact on adoption of cloud computing. International Conference on Information Society (I-Society 2014), IEEE Xplore, 92-97. https://doi.org/10.1109/i-society.2014.7009018

Pahl, C., \& Xiong, H. (2013, September 1). Migration to PaaS clouds - Migration process and architectural concerns. 2013 IEEE 7th International Symposium on the Maintenance and Evolution of Service-Oriented and Cloud-Based Systems, IEEE Xplore, 86-91. https://doi.org/10.1109/MESOCA.2013.6632740

Ramchand, K., Chhetri, M. B., \& Kowalczyk, R. (2017). Towards a Flexible Cloud Architectural Decision Framework for Diverse Application Architectures. ACIS 2017 Proceedings. https://aisel.aisnet.org/acis2017/25/

Talukder, A. K., Zimmerman, L., \& A, P. H. (2010). Cloud Economics: Principles, Costs, and Benefits. Computer Communications and Networks, 343-360. https://doi.org/10.1007/978-1-84996-241-4_20

Verdejo, A. J., \& Baars, H. (2013). Decision support for partially moving applications to the cloud: the example of business intelligence. HotTopiCS '13: Proceedings of the 2013 international workshop on Hot topics in cloud services, 35-42. https://doi.org/10.1145/2462307.2462316 
Williams, R. T. (2018). Confidence Interventions: Do They Work?. Asian Journal of Humanity, Art and Literature, 5(2), 123-134. https://doi.org/10.18034/ajhal.v5i2.536

Williams, R. T., \& Scott, C. D. (2019). The Current State of Outdoor Learning in a U.K Secondary Setting: Exploring the Benefits, Drawbacks and Recommendations. ABC Journal of Advanced Research, 109122. https://doi.org/10.18034/abcjar.v8i2.537

$--0-$ 